\title{
Sobre o limiar para a produção de pares e localização de partículas sem spin
}

(On the threshold for the pair production and localization of spinless particles)

\author{
Tatiana R. Cardoso e Antonio S. de Castro ${ }^{1}$ \\ Departamento de Física e Química, Universidade Estadual Paulista, Guaratinguetá, SP, Brasil \\ Recebido em 16/10/2006; Aceito em 13/2/2007
}

\begin{abstract}
A equação de Klein-Gordon em uma dimensão espacial é investigada com a mais geral estrutura de Lorentz para os potenciais externos. A análise do espalhamento de partículas em um potencial degrau com uma mistura arbitrária de acoplamentos vetorial e escalar revela que o acoplamento escalar contribui para aumentar o limiar da energia de produção de pares. Mostra-se ainda que a produção de pares torna-se factível somente quando o acoplamento vetorial excede o acoplamento escalar. Um aparente paradoxo relacionado com a localização de uma partícula em uma região do espaço arbitrariamente pequena, devido à presença do potencial escalar, é resolvido com a introdução do conceito de comprimento de onda Compton efetivo.

Palavras-chave: equação de Klein-Gordon, paradoxo de Klein, produção de pares, localização, comprimento de onda Compton.

The one-dimensional Klein-Gordon equation is investigated with the most general Lorentz structure for the external potentials. The analysis of the scattering of particles in a step potential with an arbitrary mixing of vector and scalar couplings reveals that the scalar coupling contributes for increasing the threshold energy for the particle-antiparticle pair production. Furthermore, it is shown that the pair production is only feasible whether the vector coupling exceeds the scalar one. An apparent paradox concerning the localization of a particle in an arbitrarily small region of space, due to the presence of the scalar coupling, is solved by introducing the concept of effective Compton wavelength.
\end{abstract}

Keywords: Klein-Gordon equation, Klein’s paradox, pair production, localization, Compton wavelength.

\section{Introdução}

A generalização da mecânica quântica que inclui a relatividade especial é necessária para a descrição de fenômenos em altas energias e também para a descrição de fenômenos em escalas de comprimentos que são menores ou comparáveis com o comprimento de onda Compton da partícula $(\lambda=\hbar /(m c))$. A generalização não é uma tarefa trivial e novos e peculiares fenômenos surgem na mecânica quântica relativística (doravante denominada MQR). Entre tais fenômenos estão a produção espontânea de pares matéria-antimatéria e a limitação para a localização de partículas. Essa limitação pode ser estimada pela observação que a máxima incerteza para o momento da partícula $\Delta p=m c$ conduz, via princípio da in-

\footnotetext{
${ }^{1}$ E-mail: castro@pesquisador.cnpq.br.

Copyright by the Sociedade Brasileira de Física. Printed in Brazil.
}

certeza de Heisenberg, à incerteza mínima na posição $\Delta x=\lambda / 2,[1,2$. Embora a MQR como modelo de partícula única, referida como formalismo de primeira quantização, não possa dar conta da completa descrição da criação de pares, ela pavimenta o caminho para o desenvolvimento da teoria quântica de campos.

As mais simples equações da MQR são a equação de Klein-Gordon $(E K G)^{2}$ e a equação de Dirac ${ }^{3}$. O spin é uma complicação adicional na MQR e, naturalmente, a EKG permite que certos aspectos da MQR possam ser analisados com um formalismo matemático mais simples e percebidos com maior transparência. A solução da equação de Dirac para o espalhamento de partículas em um potencial degrau, considerado como o componente temporal de um potencial vetorial, é bem conhecida e cristalizada em livros-texto [1-5]. Neste problema

\footnotetext{
${ }^{2}$ A EKG descreve o comportamento de bósons de spin 0. Píons e káons, por exemplo.

${ }^{3} \mathrm{~A}$ equação de Dirac descreve o comportamento de férmions de spin $1 / 2$, tais como o elétron, o neutrino, o quark, o próton e o nêutron.
} 
surge o célebre paradoxo de Klein 6 para potenciais suficientemente intensos, um fenômeno em que o coeficiente de reflexão excede a unidade e é interpretado como sendo devido à criação de pares na interface do potencial. A análise do problema consoante a EKG não foi esquecida, [5, 7-11].

Neste trabalho analisamos a EKG unidimensional com interações externas com a mais geral estrutura de Lorentz, i.e., consideramos potenciais com estrutura vetorial, com componentes espacial e temporal, acrescido de uma estrutura escalar. Em seguida exploramos as soluções para o espalhamento de partículas em um potencial degrau com acoplamento geral, por assim dizer, com uma mistura arbitrária de acoplamentos vetorial e escalar. Verificamos que tal mistura de acoplamentos conduz a resultados surpreendentes. Para além de aumentar o limiar de energia para a produção espontânea de pares, podendo até mesmo frustrar a produção ainda que os potenciais sejam extremamente fortes, a presença de um acoplamento escalar permite que uma partícula possa ser localizada em uma região do espaço arbitrariamente pequena sem ameaçar a interpretação de partícula única da EKG. A aparente violação do princípio da incerteza é remediada com a introdução do conceito de comprimento de onda Compton efetivo.

Apesar da originalidade e generalidade, este trabalho é acessível aos estudantes de graduação em física que tenham freqüentado alguns poucos meses de um curso introdutório de mecânica quântica. Dessa forma permite-se o acesso precoce de estudantes a alguns dos mais interessantes fenômenos da MQR.

\section{A equação de Klein-Gordon}

A EKG unidimensional para uma partícula livre de massa de repouso $m$ corresponde à relação energiamomento relativística $E^{2}=c^{2} p^{2}+m^{2} c^{4}$, onde a energia $E$ e o momento $p$ tornam-se operadores, $i \hbar \partial / \partial t \mathrm{e}$ $-i \hbar \partial / \partial x$ respectivamente, atuando sobre a função de onda $\Phi(x, t)$. Aqui, $c$ é a velocidade da luz e $\hbar$ é a constante de Planck $(\hbar=h /(2 \pi))$.

$\mathrm{Na}$ presença de potenciais externos a relação energia-momento torna-se

$$
\left(E-V_{t}\right)^{2}=c^{2}\left(p-\frac{V_{e}}{c}\right)^{2}+\left(m c^{2}+V_{s}\right)^{2},
$$

onde os subscritos nos termos dos potenciais denotam suas propriedades com respeito às transformações de Lorentz: $t$ e $e$ para os componentes temporal e espa- cial de um potencial vetorial ${ }^{4}$, e $s$ para um potencial escalar ${ }^{5}$.

A equação da continuidade para a EKG

$$
\frac{\partial \rho}{\partial t}+\frac{\partial J}{\partial x}=0
$$

é satisfeita com $\rho$ e $J$ definidos como

$$
\begin{aligned}
\rho & =\frac{i \hbar}{2 m c^{2}}\left(\Phi^{*} \frac{\partial \Phi}{\partial t}-\frac{\partial \Phi^{*}}{\partial t} \Phi\right)-\frac{V_{t}}{m c^{2}}|\Phi|^{2} \\
J & =\frac{\hbar}{2 i m}\left(\Phi^{*} \frac{\partial \Phi}{\partial x}-\frac{\partial \Phi^{*}}{\partial x} \Phi\right)-\frac{V_{e}}{m c}|\Phi|^{2} .
\end{aligned}
$$

Vale a pena observar o modo que os componentes do potencial vetorial participam da densidade $\rho$ e da corrente $J$, tanto quanto a ausência do potencial escalar. Observa-se também que a densidade envolve derivadas temporais, um fato relacionado com a derivada temporal de segunda ordem na EKG, e pode admitir valores negativos mesmo no caso de uma partícula livre. Assim sendo $\rho$ não pode ser interpretada como uma densidade de probabilidade. Contudo, Pauli e Weisskopf $[12$ mostraram que não há dificuldade com a interpretação da densidade e da corrente da EKG se essas grandezas forem interpretadas como densidade e corrente de carga, ao invés de densidade e corrente de probabilidade. A carga não deve ser pensada necessariamente como carga elétrica, mas como carga generalizada que satisfaz uma lei de conservação aditiva, por assim dizer que a carga de um sistema é a soma das cargas de suas partes constituintes.

Para potenciais externos independentes do tempo, a EKG admite soluções da forma

$$
\Phi(x, t)=\phi(x) e^{i \Lambda(x)} e^{-i \frac{E}{\hbar} t}
$$

onde $\phi$ obedece a uma equação similar em forma à equação de Schrödinger

$$
\begin{gathered}
-\frac{\hbar^{2}}{2 m} \frac{d^{2} \phi}{d x^{2}}+\left(\frac{V_{s}^{2}-V_{t}^{2}}{2 m c^{2}}+V_{s}+\frac{E}{m c^{2}} V_{t}\right) \phi= \\
\frac{E^{2}-m^{2} c^{4}}{2 m c^{2}} \phi
\end{gathered}
$$

$\operatorname{com} \Lambda(x)=\int^{x} d y V_{e}(y) /(\hbar c)$. A eliminação do componente espacial do potencial vetorial é equivalente a uma redefinição do operador momento. Realmente,

$$
\left(p_{o p}-\frac{V_{e}}{c}\right)^{2} \Phi=e^{i \Lambda} p_{o p}^{2} \phi .
$$

\footnotetext{
${ }^{4} \mathrm{~A}$ energia e o momento são os componentes temporal e espacial, respectivamente, da quantidade $(E / c, p)$, a qual se comporta, segundo as transformações de Lorentz, como um vetor. O potencial vetorial, com componentes $\left(V_{t}\right.$, $\left.V_{e}\right)$, é acoplado à partícula de acordo com o princípio do acoplamento mínimo, também chamado de princípio da substituição mínima, E $\rightarrow E-V_{t}$ e $p \rightarrow p-V_{e} / c$, como é habitual no caso da interação eletromagnética.

${ }^{5} \mathrm{~A}$ massa de repouso é uma quantidade invariante de Lorentz, i.e., uma quantidade escalar. O potencial escalar foi acoplado à partícula em (1) de acordo com o princípio do acoplamento mínimo $m \rightarrow m+V_{s} / c^{2}$. Esta prescrição fornece o limite não-relativístico apropriado da EKG, conforme veremos adiante, em contraste com a regra $m^{2} \rightarrow m^{2}+V_{s}^{2} / c^{4}$ empregada na Ref. [1].
} 
É agora importante perceber que há soluções de energia positiva tanto quanto soluções de energia negativa ${ }^{6}$ e que os dois possíveis sinais para $E$ implicam em duas possibilidades para a evolução temporal da função de onda. Seja como for, a energia é uma quantidade conservada. A forma da equação de autovalor (5) é preservada sob as transformações simultâneas $E \rightarrow-E$ e $V_{t} \rightarrow-V_{t}$, e isto implica que partículas e antipartículas estão sujeitas a componentes temporais de um potencial vetorial com sinais dissimilares. Como conseqüência imediata dessa covariância tem-se que, por mais estranho que possa parecer, partículas e antipartículas compartilham exatamente a mesma autofunção no caso de um potencial puramente escalar e que o espectro é disposto simetricamente em torno de $E=0$. Cargas positivas e negativas estão sujeitas a acoplamentos vetoriais (componentes temporais) de sinais contrários e igual acoplamento escalar. A interação escalar é independente da carga e assim age indiscriminadamente sobre partículas e antipartículas. Diz-se então que o potencial vetorial acopla com a carga da partícula e que o potencial escalar acopla com a massa da partícula. A densidade e a corrente correspondentes à solução expressa pela Eq. (4) tornam-se

$$
\begin{aligned}
\rho & =\frac{E-V_{t}}{m c^{2}}|\phi|^{2}, \\
J & =\frac{\hbar}{2 i m}\left(\phi^{*} \frac{\partial \phi}{\partial x}-\frac{\partial \phi^{*}}{\partial x} \phi\right) .
\end{aligned}
$$

Em virtude de $\rho$ e $J$ serem independentes do tempo, a solução (4) é dita descrever um estado estacionário. Nota-se que a densidade torna-se negativa em regiões do espaço onde $V_{t}>E$ e que o componente espacial do potencial vetorial não mais intervém na corrente.

Ademais, deve-se mencionar que a EKG reduz-se à equação de Schrödinger no limite não-relativístico $\left(E \simeq m c^{2}\right.$ e energias potenciais pequenas comparadas com $m c^{2}$ ) com $\phi$ obedecendo à equação

$$
-\frac{\hbar^{2}}{2 m} \frac{d^{2} \phi}{d x^{2}}+\left(V_{t}+V_{s}\right) \phi=\left(E-m c^{2}\right) \phi .
$$

No limite não-relativístico as naturezas de Lorentz dos potenciais não sofrem quaisquer distinções, e a densidade e a corrente reduzem-se exatamente aos valores da teoria não-relativística.

\section{A solução para um potencial degrau}

Vamos agora considerar a EKG com os potenciais externos independentes do tempo na forma de um degrau de potencial. Consideramos $V_{e}=0$, haja vista que o componente espacial do potencial vetorial contribui apenas com um fator de fase local para $\Phi(x, t)$ e não contribui para a densidade nem para a corrente. O potencial degrau é expresso como

$$
V(x)=\left\{\begin{array}{rrr}
0 & \text { para } & x<0 \\
V_{0} & \text { para } & x>0
\end{array}\right.
$$

onde $V_{0}>0$. Os potenciais vetorial e escalar são escritos como $V_{t}(x)=g_{t} V(x)$ e $V_{s}(x)=g_{s} V(x)$ de tal forma que as constantes de acoplamento estão sujeitas ao vínculo $g_{t}+g_{s}=1$, com $g_{t} \geq 0$ e $g_{s} \geq 0$.

Para $x<0$, a EKG apresenta soluções na forma de uma soma de autofunções do operador momento escrita como

$$
\phi=A_{+} e^{+i k x}+A_{-} e^{-i k x},
$$

onde

$$
k=\frac{\sqrt{E^{2}-m^{2} c^{4}}}{\hbar c} .
$$

Para $|E|>m c^{2}$, a solução expressa por (10) reverte-se em ondas planas propagando-se em ambos os sentidos do eixo $X$ com velocidade de grupd ${ }^{7}$

$$
v_{g}=\frac{1}{\hbar} \frac{d E}{d k},
$$

igual à velocidade clássica da partícula. Se escolhermos partículas incidindo sobre a barreira de potencial $\left(E>m c^{2}\right)$ teremos que $A_{+} e^{+i k x}$ descreve partículas incidentes $\left(v_{g}=c^{2} \hbar k / E>0\right)$, enquanto $A_{-} e^{-i k x}$ descreve partículas refletidas $\left(v_{g}=-c^{2} \hbar k / E<0\right)$. A corrente nesta região do espaço, correspondendo a $\phi$ dada por (10), é expressa por

$$
J=\frac{\hbar k}{m}\left(\left|A_{+}\right|^{2}-\left|A_{-}\right|^{2}\right) .
$$

Observe que a relação $J=\rho v_{g}$ mantém-se tanto para a onda incidente quanto para a onda refletida, pois

$$
\rho=\frac{E}{m c^{2}}|\phi|^{2}>0
$$

Por outro lado, para $x>0$ devemos ter $v_{g} \geq 0$ de forma que a solução nesta região do espaço descreve uma onda evanescente ou uma onda progressiva que se afasta da interface do potencial. A solução geral tem a forma

$$
\phi=B_{+} e^{+i \kappa x}+B_{-} e^{-i \kappa x},
$$

onde

$$
\kappa=\frac{\sqrt{\left(E-g_{t} V_{0}\right)^{2}-\left(m c^{2}+g_{s} V_{0}\right)^{2}}}{\hbar c} .
$$

Por causa da dupla possibilidade de sinais para a energia de um estado estacionário, a solução $B_{-} e^{-i \kappa x}$ não pode ser descartada a priori. De fato, pode-se depreender da Eq. (4) que esta parcela pode vir a descrever uma onda progressiva com energia negativa e velocidade de fase $v_{f}=|E| /(\hbar \kappa)>0$. Percebe-se claramente que podemos segregar três classes distintas de soluções:

\footnotetext{
${ }^{6}$ As soluções de energia positiva e negativa são assossiadas com partículas e antipartículas, respectivamente.

${ }^{7}$ Veja, e.g., Refs. [1] e [5].
} 
- Classe A. Para $V_{0}<E-m c^{2}$ temos que $\kappa \in \mathbb{R}$ e a solução que descreve ondas planas propagando-se no sentido positivo do eixo $X$ com velocidade de grupo

$$
v_{g}=\frac{c^{2} \hbar \kappa}{E-g_{t} V_{0}},
$$

é possível somente se $B_{-}=0$. Neste caso, a densidade e a corrente são dadas por

$$
\rho=\frac{E-g_{t} V_{0}}{m c^{2}}\left|B_{+}\right|^{2} \quad \text { e } \quad J=\frac{\hbar \kappa}{m}\left|B_{+}\right|^{2} .
$$

- Classe B. Para $E-m c^{2}<V_{0}<V_{c}$, onde

$$
V_{c}=\left\{\begin{array}{cl}
\frac{E+m c^{2}}{2 g_{t}-1} & \text { para } g_{t}>\frac{1}{2} \\
\infty & \text { para } g_{t} \leq \frac{1}{2}
\end{array}\right.
$$

temos que $\kappa=i|\kappa|$ de forma que a Eq. (15), com $B_{-}=0, \frac{8}{8}$ descreve uma onda evanescente. Neste caso

$$
\rho=\frac{E-g_{t} V_{0}}{m c^{2}}\left|B_{+}\right|^{2} e^{-2|\kappa| x}, \quad \text { e } \quad J=0 .
$$

- Classe C. $V_{0}>V_{c}$, com $V_{c}$ concebido na classe B, surge mais uma vez a possibilidade de propagação no sentido positivo do eixo $X$, desta feita com $B_{+}=0$, com velocidade de grupo

$$
v_{g}=\frac{c^{2} \hbar \kappa}{g_{t} V_{0}-E} .
$$

Nesta circunstância em que o acoplamento vetorial excede o acoplamento escalar nos defrontamos com um caso bizarro, pois tanto a densidade quanto a corrente são quantidades negativas, viz

$$
\rho=\frac{E-g_{t} V_{0}}{m c^{2}}\left|B_{-}\right|^{2}, \quad \text { e } \quad J=-\frac{\hbar \kappa}{m}\left|B_{-}\right|^{2} .
$$

A mantença da relação $J=\rho v_{g}$, contudo, é uma licença para interpretar $B_{-} e^{-i \kappa x}$ a descrever a propagação, no sentido positivo do eixo $X$, de partículas com carga de sinal contrário ao das partículas incidentes. Esta interpretação é consistente se as partículas propagando-se nessa região têm energia $-E$ e estão sob a influência de um potencial vetorial $-g_{t} V_{0}$. Quer dizer, então, que a onda progressiva descreve, de fato, a propagação de antipartículas no sentido positivo do eixo $X \underline{9}^{9}$

\subsection{Os coeficientes de reflexão e transmissão}

Não obstante a descontinuidade do potencial em $x=0$, a autofunção e sua derivada primeira são funções contínuas. ${ }^{[10}$ A demanda por continuidade de $\phi$ e $d \phi / d x$ fixa as amplitudes de onda em termos da amplitude da onda incidente $A_{+}$, viz

$$
\begin{gathered}
\frac{A_{-}}{A_{+}}=\left\{\begin{array}{cc}
\frac{k-\kappa}{k+\kappa} & \text { para a classe A } \\
\frac{(k-i|\kappa|)^{2}}{k^{2}+|\kappa|^{2}} & \text { para a classe B } \\
\frac{k+\kappa}{k-\kappa} & \text { para a classe C }
\end{array}\right. \\
\frac{B_{+}}{A_{+}}=\left\{\begin{array}{cc}
\frac{2 k}{k+\kappa} & \text { para a classe A } \\
\frac{2 k(k-i|\kappa|)}{k^{2}+|\kappa|^{2}} & \text { para a classe B } \\
0 & \text { para a classe C }
\end{array}\right. \\
\frac{B_{-}}{A_{+}}=\left\{\begin{array}{cc}
0 & \text { para a classe A } \\
0 & \text { para a classe B } \\
\frac{2 k}{k-\kappa} & \text { para a classe C. }
\end{array}\right.
\end{gathered}
$$

Agora focalizamos nossa atenção na determinação dos coeficientes de reflexão $R$ e transmissão $T$. O coeficiente de reflexão (transmissão) é definido como a razão entre as correntes refletida (transmitida) e incidente. Haja vista que $\partial \rho / \partial t=0$ para estados estacionários, temos que a corrente é independente de $x$. Usando este fato obtemos prontamente que

$$
R=\frac{\left|A_{-}\right|^{2}}{\left|A_{+}\right|^{2}}=\left\{\begin{array}{cc}
\left(\frac{k-\kappa}{k+\kappa}\right)^{2} & \text { para a classe A } \\
1 & \text { para a classe B } \\
\left(\frac{k+\kappa}{k-\kappa}\right)^{2} & \text { para a classe C. }
\end{array}\right.
$$

$$
T=\left\{\begin{array}{cl}
\frac{\kappa}{k} \frac{\left|B_{+}\right|^{2}}{\left|A_{+}\right|^{2}}=\frac{4 k \kappa}{(k+\kappa)^{2}} & \text { para a classe A } \\
0 & \text { para a classe B } \\
-\frac{\kappa}{k} \mid \frac{\left.B_{-}\right|^{2}}{\left|A_{+}\right|^{2}}=-\frac{4 k \kappa}{(k-\kappa)^{2}} & \text { para a classe C. }
\end{array}\right.
$$

Em todas as classes temos que $R+T=1$, como deve ser. Entretanto, a classe C apresenta $R>1$, o aludido paradoxo de Klein, implicando que mais partículas são refletidas na barreira de potencial que aquelas incidentes. Deve ser assim porque, conforme vimos anteriormente, o componente vetorial da barreira de potencial estimula a produção de antipartículas em $x=0$.

\footnotetext{
${ }^{8} \mathrm{~A}$ condição $B_{-}=0$ é necessária para que a densidade seja finita quando $x \rightarrow+\infty$.

${ }^{9}$ Note que partícula e antipartícula têm massas iguais.

${ }^{10}$ Esta conclusão, válida para potenciais com descontinuidades finitas, pode ser obtida pela integração da Eq. (5) entre $-\varepsilon$ e $+\varepsilon$ no limite $\varepsilon \rightarrow 0$. Pode-se verificar, pelo mesmo procedimento, que apenas as autofunções são contínuas quando as descontinuidades dos potenciais são infinitas.
} 
Em virtude da conservação da carga há, em verdade, a criação de pares partícula-antipartícula e, como o potencial vetorial em $x>0$ é repulsivo para partículas, elas serão necessariamente refletidas. Não apenas a carga é conservada. Visto que os pares produzidos em $x=0$ têm energias de sinais contrários, conclui-se que a energia também é uma quantidade conservada no processo de criação de pares.

\subsection{O limiar para a produção de pares}

Da discussão relacionada com as classes B e C, observase que o limiar para a produção de pares é dado por $V_{c}$. Donde torna-se evidente que o acoplamento escalar resulta no aumento da energia mínima necessária para a criação de pares partícula-antipartícula. O valor mínimo do limiar $\left(V_{0}=2 m c^{2}\right)$ ocorre quando o acoplamento é puramente vetorial $\left(g_{t}=1\right)$. A adição de um contaminante escalar contribui para aumentar o valor do limiar, o qual, surpreendentemente, torna-se infinito já para uma mistura meio a meio de acoplamentos. Deste modo, a produção de pares não é factível se o acoplamento vetorial não exceder o acoplamento escalar, ainda que o potencial $V_{0}$ seja extremamente forte.

Pode-se interpretar a possibilidade de propagação de antipartículas além da barreira de potencial como sendo devido ao fato que cada antipartícula está sujeita a um potencial efetivo dado por $\left(g_{s}-g_{t}\right) V_{0}$, destarte se $g_{t}>1 / 2$ a antipartícula terá uma energia disponível (energia de repouso mais energia cinética) expressa por $\left(2 g_{t}-1\right) V_{0}-E$, donde se conclui sobre a energia do limiar da produção de pares. Pode-se afirmar ainda que as partículas estão sob a influência de um potencial degrau ascendente de altura $V_{0}=\left(g_{s}+g_{t}\right) V_{0}$, e que as antipartículas estão sujeitas a um potencial degrau efetivo de altura $\left(g_{s}-g_{t}\right) V_{0}$, um degrau ascendente (repulsivo) se $g_{t}<1 / 2$ e descendente (atrativo) se $g_{t}>1 / 2$.

\subsection{A penetração na região classicamente proibida}

Investigamos agora o efeito da onda evanescente em $x>0$, relacionado com a classe B. Neste caso, o estado estacionário além da barreira de potencial é descrito pela autofunção $\phi=B_{+} e^{-|\kappa| x}$, de modo que a incerteza na posição, estimada como sendo o valor de $x$ que torna a densidade igual a $1 / e$ de seu valor em $x=0$, redunda em $\Delta x=1 /(2|\kappa|)$, como acontece na teoria quântica não-relativística. Entretanto, contrariamente à previsão da teoria não-relativística, $\Delta x$ apresenta o valor mínimo

$$
(\Delta x)_{\min }=\frac{\hbar}{2\left(m c+g_{s} V_{0} / c\right)},
$$

quando $V_{0}$ torna-se igual a

$$
V_{m}=\frac{E}{g_{t}} .
$$

Por meio desta última expressão vemos que $(\Delta x)_{\min }=\lambda / 2$ no caso de um potencial vetorial puro $\left(g_{s}=0\right)$, em harmonia com o príncipio da incerteza. Contudo, podemos concluir que $(\Delta x)_{\min }<\lambda / 2$ no caso de um potencial vetorial contaminado com algum acoplamento escalar. À primeira vista isto parece um resultado desatroso por violar o princípio da incerteza de Heisenberg. Liberta-se desta danação considerandose que o componente escalar do potencial contribui para alterar a massa da partícula. Realmente, definindo a massa efetiva como $m_{\mathrm{ef}}=m+g_{s} V_{0} / c^{2}$ segue-se imediatamente que $(\Delta x)_{\min }=\lambda_{\mathrm{ef}} / 2 \mathrm{e}(\Delta p)_{\max }=m_{\mathrm{ef}} c$, onde o comprimento de onda Compton efetivo é definido como $\lambda_{\mathrm{ef}}=\hbar /\left(m_{\mathrm{ef}} c\right)$.

\section{Conclusão}

Exploramos a EKG em uma dimensão espacial por motivos de simplicidade. Consideramos potenciais externos com a mais geral estrutura de Lorentz e mostramos que, se a interação escalar é acoplada adequadamente, a EKG independente do tempo reduz-se à equação de Schrödinger independente do tempo no limite não-relativístico.

A análise do potencial degrau com uma mistura arbitrária de acoplamentos vetorial e escalar mostrou-se muito profícua. Três classes de soluções foram discernidas. Em todas essas três classes, o acoplamento escalar não desempenha papel explícito na determinação da velocidade de grupo, e nenhum papel na determinação da densidade e da corrente.

A mistura arbitrária de acoplamentos no potencial degrau desvelou a inexeqüibilidade do mecanismo da produção espontânea de pares no caso em que $g_{t} \leq 1 / 2$, tanto quanto o aumento do limiar da energia de produção no caso em que $g_{t}>1 / 2$. Outrossim, a presença de um acoplamento escalar revelou a possibilidade de localizar partículas em regiões do espaço arbitrariamente pequenas. Com efeito, a presença de um acoplamento escalar, por menor que seja, conduz a $(\Delta x)_{\min } \rightarrow 0$ quando $V_{0} \rightarrow \infty$ sem que haja qualquer chance para a produção de pares na interface dos potenciais. Isto dito tendo em vista que $V_{m}$, o potencial que minimiza a incerteza na posição, é sempre menor que $V_{c}$, o potencial do limiar da produção espontânea de pares.

O limite não-relativístico da EKG expresso pela Eq. (8), ou seja a equação de Schrödinger com energia de ligação $E-m c^{2}$, não diferencia o acoplamento vetorial do acoplamento escalar e pressupõe que $V_{0}<<m c^{2}$. Portanto, conclui-se seguramente que não há produção de pares $\left(\left(V_{c}\right)_{\min }=2 m c^{2}\right)$ nem incerteza mínima na posição $\left(\left(V_{m}\right)_{\min }=m c^{2}\right)$ no regime não-relativístico da EKG, como é esperado.

Naturalmente, os coeficientes de reflexão e transmissão para a classe de soluções que envolve a criação de pares foram determinados de maneira aproximada, 
porquanto descuidou-se da interação interna entre partículas e antipartículas.

Finalmente, ainda que haja interações externas extremamente fortes, a inviabilidade do mecanismo de produção de pares no caso em que o acoplamento escalar excede o acoplamento vetorial parece preservar a interpretação do modelo de partícula única da EKG. Entretanto, quando as condições favoráveis ao mecanismo de produção de pares entram em cena, já não se pode mais esperar que o formalismo de primeira quantização seja satisfatório. Ainda que tais condições não se manifestem, resta perguntar qual o papel dos estados associados com as antipartículas. Mesmo na ausência de potenciais externos, qual o mecanismo que evita que haja transições entre o estados de energia positiva pertencentes ao continuum entre $+m c^{2} \mathrm{e}+\infty$, e os estados de energia negativa pertencentes ao continuum entre $-m c^{2}$ e $-\infty$ ? Eis aqui exemplos de perguntas que encontram respostas satisfatórias somente no formalismo da segunda quantização da teoria.

\section{Agradecimentos:}

Os autores são gratos ao CNPq e à FAPESP pelo apoio financeiro.

\section{Referências}

[1] W. Greiner, Relativistic Quantum Mechanics, Wave Equations (Springer-Verlag, Berlim, 1990).

[2] P. Strange, Relativistic Quantum Mechanics with Applications in Condensed Matter and Atomic Physics (Cambridge University Press, Cambridge, 1998).

[3] J.D. Bjorken and S.D. Drell, Relativistic Quantum Mechanics (McGraw-Hill, Nova Iorque, 1964).

[4] J.J. Sakurai, Advanced Quantum Mechanics (AddisonWesley, Reading, 1967).

[5] F. Gross, Relativistic Quantum Mechanics and Field Theory (Wiley, Nova Iorque, 1993).

[6] O. Klein, Z. Phys. 53, 157 (1929).

[7] R.G. Winter, Am. J. Phys. 27, 355 (1959).

[8] M.G. Fu and E. Furlani, Am. J. Phys. 50, 545 (1982).

[9] B.R. Holstein, A. J. Phys. 66, 507 (1998).

[10] J.-J. Ni, W. Zhou and J. Yan, Klein Paradox and Antiparticle, arXiv: quant-ph/9905044.

[11] J. Villavicencio, J. Phys. A 33, 6061 (2000).

[12] W. Pauli and V.F. Weisskopf, Helv. Phys. Acta 1, 709 (1934). 